Volume 8. No. 10, October 2020

International Journal of Emerging Trends in Engineering Research

Available Online at http://www.warse.org/IJETER/static/pdf/file/ijeter1058102020.pdf

https://doi.org/10.30534/ijeter/2020/1058102020

\title{
Top approaches to abstractive text summarization: A survey
}

\section{Deepak Sahoo ${ }^{1}$, Chayan Paul ${ }^{2}$, Praveen Tumuluru ${ }^{3}$}

${ }^{1}$ Dept. of CSE, Koneru Lakshmaiah Education Foundation, Vaddeswaram, India, deepsahoo@gmil.com

${ }^{2}$ Dept. of CSE, Koneru Lakshmaiah Education Foundation, Vaddeswaram, India, chayan@ kluniversity.in

${ }^{3}$ Dept. of CSE, Koneru Lakshmaiah Education Foundation, Vaddeswaram, India, praveenluru@kluniversity.in

\begin{abstract}
We are in the age of information overload where a high volume of data and information is present over the internet. There are different types of text mining tasks are available to extract the appropriate data as per the user need. In text mining, summarization is a task where the gist of the source text is generated by the system. Extractive and abstractive are the two variants of text summarization based on context. Extractive approach requires less engineering and linguistic effort whereas abstractive text summarization is still a demanding task among natural language processing researchers. Abstractive text summarization system understands, interpret the original text and presents the text in new form therefore abstractive summarization require more engineering and linguistic efforts.
\end{abstract}

Key words: Abstractive summarization, Extractive summarization, Text mining, Text summarization.

\section{INTRODUCTION TO TEXT MINING}

In text mining [1], unstructured or semi-structured textual data is processed to find useful numerical indices that can be used by the various data mining algorithms to extract meaningfull informatin from the text. In general terms text mining will "turn text into meaningful indices" and used in different analyses such as predictive data mining applicatons, clustering and classification. These techniques are discussed and explained in the work by Manning and Schütze [2]. The typical tasks of text mining shown in Figure 1 can be given as document clustering, organization, classification, and Information extraction.

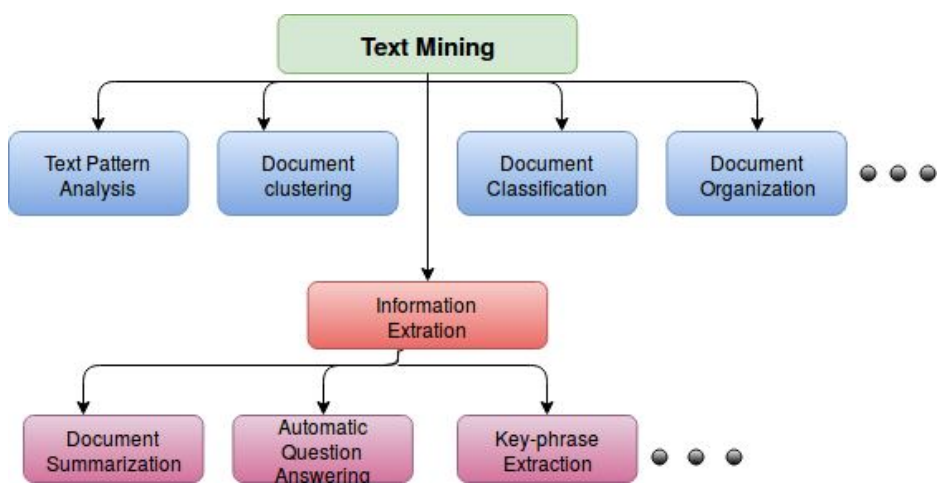

Figure 1: Text mining approaches

\section{DOCUMENT SUMMARIZATION}

Text summarization is an important tool to summarize large text because summarizing a large text manually is a tough task. The purpose of automatic text summarizaton is to present shorten and non-redundant verrsion of source text without negotiating the general meaning of the text.

This summarization task can be classified based on form, dimension and context given in Figure 2. An extractive summarization method $[3,4,5,6]$ has two parts, extracting most salient sentences from source text and fuseing them into shorter form. The most infromative sentences are extracted based on linguistic and statistical attributes of sentences.

In the abstractive approach, it is not only extracting the sentences but the system needs to understand the meaning, merge the sentences, add new words, remove unnecessary words and generate a new sentence. Therefore, abstractive summarization is challenging that require more linguistic effort compared to extractive summarization.

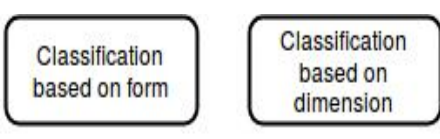

Classification based on context

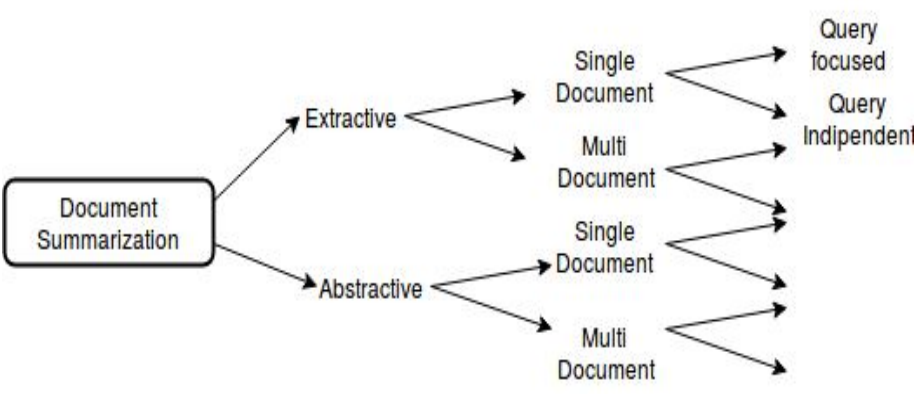

Figure 2: Classification of text summarization

Abstractive and extractive summarization can be multi document or single document summarization. if the system takes one document as input then it is a single document extractive/abstractive summarization. If the system takes more than one document as input then it is considered as multidocument extractive/abstractive summarization. Again the single/multi-document extractive/abstractive 
summarization can be query focused or query independent. If the summary is generated based on a user query then its query-focused summarization or query independent summarization.

\subsection{Back ground of extractive summary}

A lot of work has been done and many approaches are used to extract best and informative sentences from the document since its inception in 1958 [7] where a sentence of a document can be mapped as a function of high-frequency words. In addition to standard keywords, the summarization system [8] used below three techniques to calculate weight of the sentence.

1. Cue technique: In this technique, the weight of a sentence is assessed by the occurance of certain cue words.

2. Title technique: In this technique, the weight of the sentence is computed taking the sum of all the content words present in the title.

3. Location technique: This technique assumes that, more relevant sentences are present in initial positions of the text.

Text extraction system ANES [9] is an domain independent news summarization sustem has following four components.

1. Analysis of corpus: tf*idf-weights of all terms are calculated in this component.

2. Selection of key words (Statistical approach): the top tf*id weighted words added with headlinewords.

3. Sentence weight: summing the weight of all the words in the sentence and some other factors like relative position of sentence.

4. Sentence selection: Rank the sentences based on weight and choosing top ranked sentence.

The trainable document summarizer [10] extracts roughly about twenty percent of the original text, based on following weighting heuristics.

1. Length of sentence: Sentence length should be greater than five words.

2. Paragraph: sentence location in a paragraph.

3. Fixed phrase: sentences containing manually chosen phrases.
4. Thematic word feature: words that occurs most frequently (except stops) are called as thematic words. Sentence score is a function of frequencies of these thematic words.

Other most important techniques like inter-document link generation [11], rhetorical structures of texts [12], lexical chain and WordNet [13] are used to generate the summary of text. MEAD [14] a multi-document summarizer creates the clusters of related sentences based on topic detection and generates the summay from cluster centroids.

\subsection{Drawbacks of extractive summary}

In extractive summary key sentences or passages are extracted from a large text, based on statistical analysis of individual or group of features such as cue words, location and word/phrase frequency to locate and extract the best sentences. The "most important" content is considered as the "most frequent" or "most favorably positioned" content but the issues with extractive summary $[15,16]$ are:

1. Generally extracted sentences are longer than the average sentence length. Therefore, some unnecessary segments are also included in the summary, consuming space.

2. It is difficult to capture important and relevant informaition as it is spread across the sentences.

3. Difficult to present (sometimes may not be) inconsistent information correctly.

4. In extractive summary "dangling" anaphora is a frequent and serious issue. Let two sentences have an anaphoric relation in a document and during extraction sentence containing the pronoun is selected but the sentence containing the propernoun/common noun that refers to that pronoun is not selected then the overall summary becomes incoherent.

\subsection{Abstractive summary and challenges}

It is observed from the background study that there is less opportunity to explore in extractive approach which requires less linguistic effort but the abstractive summary is still a challenging area to work on that require a grate many linguistic effort. Abstractive summarization system tries to realize the theme of the document and express it in natural language. This requires paraphrasing, removal of unnecessary words, and addition of new words.

The biggest challehge for the abstractive summary is to understand the text and represent it in natural language. In a specific domain, it may be feasible to come up with an 
appropriate structures, but it is difficult and challenging for open domain semantic analysis.

\section{APPROACHES TO ABSTRACTIVE SUMMARY}

In this section, we mostly discussed abstract summarization. Different researchers follow a different approach to generate an abstract summary of a text document. The important approaches to generate abstract summary are given below.

\section{Template based approach}

\section{Graph Based approach}

3. Discourse and rhetorical based approach

4. Structural approach

5. Statistical and structural approach

6. Hybrid approach

7. Optimization based approach

8. Word association approach

9. Machine learning approach

10. Deep learning approach

\subsection{Template based approaches}

Generally template based approach creates templates to generate summary of a document. In 1998, Radev et al.[17] proposed a summarization system named as SUMMONS which is a linguistic and conceptual summarization system. To generate summary SMMONS takes a set of templates.

The proposed work [18] is baes on fully abstractive approach. This is an improvement over the previously proposed work by the same authors Genest et al.[19, 20] based on abstraction schemes. The abstraction schemes are nothing but templates designed for each word in criminal domain.

Advantages: This approach is a good approach to generate summary of documents of a specific domain.

Disadvantage: Template based systems are not generic in nature and it is restricted to a specific domain. For each domain we have to define different schemes or templates for different domains to get the summary of a document. The issue with the system is that creating schemes or templates even for a domain is not scalable.

\subsection{Graph based approaches}

Graph is an important technique where many applications can be modeled as a graph based [21, 22, 23] problem. In this approach, each sentence or word represented as node in the graph and the edge represents the strength of relationship between two nodes. Then some technique is applied to generate summary.

The proposed graph based work [24, 25], first clustered the sentences then to find the difference and commonality between the clusters multiple-sequence alignment (MSA) technique is used. To calculate MSA score only word level similarity is considered but the semantics between the sentences has not been taken into account.

Opoinosis, one of the important graph-based model proposed by Ganesan et al. (2010) [26] generates concise, a non-redundant abstract summary of opinions or reviews given by the users regarding a product. The issue with this system is that it does not validate the gramatical correctness of the newly generated sentence.

Katja Filippova [27] proposed a simple, robust and graphbased model which is almost similar to Opinosis [25] they claim that this is the first technique that requires neither a parser, nor handcrafted rules, nor a language model to generate a grammatically correct sentence.

Other important abstractive summarization approach are [28, 29] use Rich Semantic Graph (RSG) and vertex constrained shortest path scheme to generate the summary,

Fei Liu et al. [30] conduct the research to explore the viability of an abstractive summarization system based on transformations of semantic representations such as the Abstract Meaning Representation (AMR; Banarescu et al.) [31]. In this work, the three steps of summarization are: (1)Creating AMR graphs for each sentence using parser. (2)Combining all the graths into a single summary AMR graph . (3) Generate the text from summary AMR graph.

Advantages: This is one of the popular approach to generate summary. Using graph based approach is used to identify the N-gram phrases. This approach is very useful to identify a valid start and end word of a sentence.

Disadvantages: Almost all the graph based techniques that are applied to generate a summary of a document or a group of reviews on a product try to find valid paths(Valid informative sentence) joining the connected nodes. It is required to validate the grammatical correctness of the generated sentence. Some systems do not validate the generated systems; some systems use syntactical analyzer to validate the sentence which is very costly in terms of execution time. 


\subsection{Discourse and rhetorical approach}

In this approach, researchers use discourse parser to generate discourse tree of the sentences and use the discourse structure to find relationship between sentences. Then find strongly related sentences for summarization. Some researchers use discourse parser to generate aspect hierarchy tree of product reviews to generate generalized message about the product.

The System SEA [32] takes a hand-crafted feature set; then natural language summary is generated using the concept content stuucturing, lexical selection, sentence planing and realization given by Reiter et al. [33].

Rhetorical structure [34, 35, 36, 37, 38, 39], is an important concept used for abstractive summarization. However, it need to parse the text fully, which is a time consuming and complex process.

Advantages: To generate the summary this approach considers the context i.e it finds the relationship with its near by sentences in the document.

Disadvantages: This approach requires complete rhetorical parsing of sentences to understand the rhetorical structure of the sentences. If the corpus is huge then this system is not recommended as full parsing takes lots of time.

\subsection{Structural approach}

This approach relies mostly on the grammatical structure of a sentence. In the system [43], the sentence passed through a syntactic parser and the output of the parser taken by the system and regenerates the sentence using a NLG (Natural language generation) engine. Summary is generated from the selected regenerated sentences based on the document frequency of contained words.

The work presented in $[44,45]$ generates abstract summart from abstract representation of the source document not from the sentences. This abstract representation depends on the Information Items (INIT), the smallest coherenet information in the text.

Advantage: The grammatical correctness of the summary generated by this approach is better. This approach performs better in single document summarization compared to multidocument summarization.

Disadvantages: As discussed this approach based on syntactic analysis of sentence therefore these systems rely heavily on syntactic analyzer or syntactic parser. In this approach each sentence need to be passed through the syntactic analyzer in a document. For multi-document summarization its not a good idea to to use structural approach.

\subsection{Statistical and structural approach}

This is a very popular approach to generate summary. The basic idea of the approach [46] is to extract important sentences based on the words frequencies, position in the sentence and syntactic information of the words. The assumptions of this technique is as follows:

1. The sentences that are related closely to theme of the text occur frequently in the text.

2. The sentences that are related to the topic often occur in some particular structure.

Advantage: This is a very simple approach to generate the summary.

Disadvantages: This approach is more extractive than abstractive.

\subsection{Hybrid approach}

In this approach, bests of more than one technique or approach are merged to generate the summary. The proposed work Starlet-H [40] is a hybrid approach that takes bests of abstractive and extractive approach. The salient qutes are filtered using using extractive technique and makes abstract summay using Rhetorical Structure Theory (RST) [41].

The proposed method by Le, H. T. et al. [43] generates abstarct summary using discourse rules, syntactic constraints, and word graph. In this approach sentences are created from keywords using discourse rule and syntactic constraints. Word graph is used to combine several sentences into one.

Advantage: Advantage of this system is that it uses the best of the of the different approach and put it in a single pipeline therefore the quality of the summary is somehow better.

Disadvantages: Although the hybrid approach takes the best of other approach still it relies on same discourse parser, syntactic analyzer, sentence parser and word graph etc. therefore the system complexity and the execution time is a somehow increased.

\subsection{Optimization based approach}

In this approach, the summarization problem is framed as a maximization or minimization problem. The proposed work $[47,48]$ has two steps, in step one facts and concepts are 
created using the phrases from the input document. In step two, new sentences are created by selecting and fuseing informative phrases while satisfy the sentence construction constraints.

Another important work of this approach AdaSum [49] that assumes topic representation and summary can be boosted manually. It aims to optimize the topic representation and extract summary simultaneously.

Advantage: In text summarization it is required to identify noun phrase, verb phrase and talked about topic in a document. Optimization technique frames the summarization problem as a maximization problem to identify noun phrase, verb phrase and topics.

Disadvantages: The problem with the optimization technique is that it uses external solver to solve the maximization problem.

\subsection{Word association approach}

The proposed method [55] for document summarizatioversion, aims to generate an abstract version of a news story. This models takes a document ' $D$ ' and a background corpus ' $\mathrm{B}$ ' as input. It has two parts, computating document-specific word associations and selecting the sentences with strong word associations.

Disadvantages: Needs a big corpus in backend to get the word association to select the sentence.

\subsection{Machine learning based approach}

With advancement in technology (i.e Machine learning, Deep learning), there is an increase in active research in abstractive summarization among the researchers. Machine learning is applied in various area $[50,51,52,53,54]$ and it is giving good results. Following are some important works in abstractive summarization using machine learning.
The proposed fully data-driven approach [56] to abstractive sentence summarization utilizes a local attention-based model that generates each word of the summary conditioned on the input sentence. The abstractive text summarization using Attentional Encoder-Decoder Recurrent Neural Networks $[56,57]$ and show that they achieve state-of-theart performance on two different corpora.

\subsection{Deep learning based approach}

Now-a-days deep-learning techniques are used in many research area $[58,59,60,67,68]$ to get more accuracy and improved result. Deep-learning technique is used to improve the quality of abstractive summary proposed in $[65,66]$. The authors follow a pyramid structure given in Figure 3 to extract knowledge from a text document. Deep-learning technique is used in one or two stages in pyramid structure.

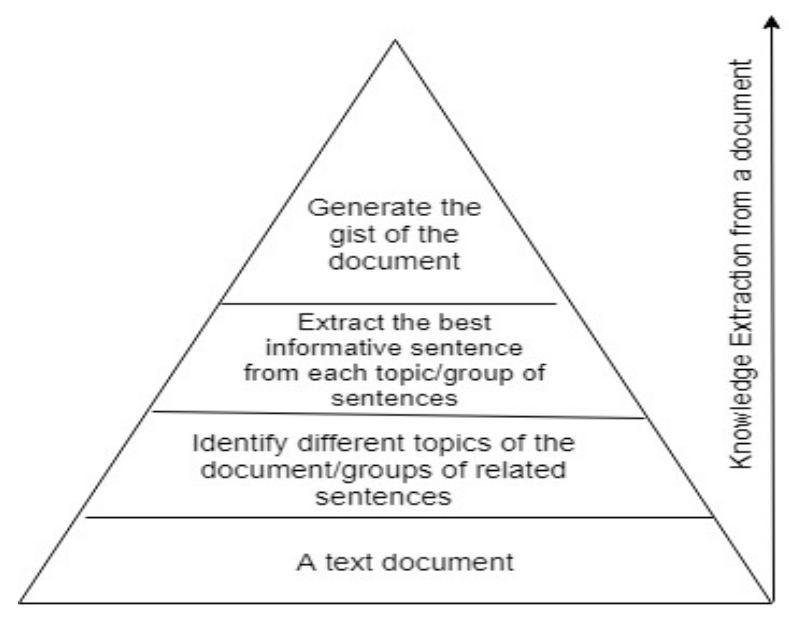

Figure 3. Pyramid approach to knowledge extraction

The summary of some relevant abstractive system elaborated in the table 1.

Table 1: Some selected works on abstractive summarization

\begin{tabular}{|l|l|l|l|l|l|l|}
\hline $\begin{array}{l}\text { Sl. } \\
\text { No }\end{array}$ & Author\& Title & $\begin{array}{l}\text { Approach } \\
\text { /Technique } \\
\text { \& Input }\end{array}$ & Dataset & $\begin{array}{l}\text { Evaluation } \\
\text { Metric }\end{array}$ & Accuracy & $\begin{array}{l}\text { Limitation and } \\
\text { Future Work }\end{array}$ \\
\hline 1 & $\begin{array}{l}\text { Zhang, J. et. al. } \\
\text { (2008), “AdaSum: An } \\
\text { Adaptive Model } \\
\text { for Summarization" }\end{array}$ & $\begin{array}{l}\text { Optimization } \\
\text { Based Ap- } \\
\text { proach \& multi } \\
\text { document }\end{array}$ & DUC2007 & $\begin{array}{l}\text { Rouge-one } \\
\text { Rouge SU4 }\end{array}$ & $\begin{array}{l}\text { ROUGE-2 } \\
=0.1172 \\
\text { ROUGE-SU4 }= \\
0.1692\end{array}$ & \\
\hline 2 & $\begin{array}{l}\text { Bing, L. Et al. (2015), } \\
\text { "Abstractive } \\
\text { Multi-document }\end{array}$ & $\begin{array}{l}\text { Optimization } \\
\text { Based Approach } \\
\text { \& multi docu- }\end{array}$ & TAC 2011 & Pyramid score & $\begin{array}{l}\text { Pyramid score }= \\
0.905\end{array}$ & \\
\hline
\end{tabular}


Deepak Sahoo et al., International Journal of Emerging Trends in Engineering Research, 8(10), October 2020, 7294 - 7304

\begin{tabular}{|c|c|c|c|c|c|c|}
\hline & $\begin{array}{l}\text { Summarization } \\
\text { via Phrase Selection } \\
\text { and Merging" }\end{array}$ & men & & & & \\
\hline 3 & $\begin{array}{l}\text { Gross O. Et } \\
\text { al. (2014), "Document } \\
\text { Summarization Based } \\
\text { on Word } \\
\text { Associations" }\end{array}$ & $\begin{array}{l}\text { Word Association } \\
\& \\
\text { Single document }\end{array}$ & DUC 2007 & Rouge Score & $\begin{array}{l}\text { Rouge-one }= \\
0.424 \text { Rouge-two } \\
=0.104 \text { Rouge- } 3= \\
0.036 \\
\text { Rouge- } \mathrm{L}=0.384\end{array}$ & \\
\hline 4 & $\begin{array}{l}\text { Rush, A. M. Et } \\
\text { al. (2015), "A Neural } \\
\text { Attention Model } \\
\text { for Abstractive } \\
\text { Sentence } \\
\text { Summarization" }\end{array}$ & $\begin{array}{l}\text { Machine-learning } \\
\text { Based } \\
\text { Approach \& } \\
\text { Single sentence }\end{array}$ & $\begin{array}{l}\text { DUC2004 } \\
\text { and } \\
\text { Gigaword }\end{array}$ & Rouge Score & $\begin{array}{l}(\text { Using DUC }) \\
\text { Rouge-one=28.18 } \\
\text { Rouge-two = } 8.49 \\
\text { Rouge-L= } 23.81\end{array}$ & \\
\hline 5 & $\begin{array}{l}\text { Chopra, S. Et al. } \\
\text { (2016), "Abstractive } \\
\text { Sentence } \\
\text { Summarization with } \\
\text { Attentive Recurrent } \\
\text { Neural Networks" }\end{array}$ & $\begin{array}{l}\text { Machine-learning } \\
\text { Based } \\
\text { Approach \& } \\
\text { Single Sentence }\end{array}$ & $\begin{array}{l}\text { DUC2004 } \\
\text { and } \\
\text { Gigaword }\end{array}$ & Rouge Score & $\begin{array}{l}\text { (Using gigaword) } \\
\text { Rouge-one = } \\
33.78 \\
\text { Rouge-two }= \\
15.97 \\
\text { Rouge-L = 31.15 } \\
\text { (Using DUC2004) } \\
\text { Rouge-one = } \\
28.97 \\
\text { Rouge-two = 8.26 } \\
\text { Rouge-L }=24.06\end{array}$ & \\
\hline 6 & $\begin{array}{l}\text { Nallapati, R. Et } \\
\text { al. (2016), Abstractive } \\
\text { Text Summarization } \\
\text { using Sequence- } \\
\text { to-sequence RNNs } \\
\text { and Beyond }\end{array}$ & $\begin{array}{l}\text { Machine-learning } \\
\text { Based } \\
\text { Approach \& multi } \\
\text { sentence }\end{array}$ & DUC2003 & Rouge Score & $\begin{array}{l}\text { Rouge-one }=28.97 \\
\text { Rouge-two }=9.46 \\
\text { Rouge-L }=25.24\end{array}$ & \\
\hline 7 & $\begin{array}{l}\text { Fabbrizio et al. } \\
\text { (2014), "A Hybrid } \\
\text { Approach to Multi- } \\
\text { document } \\
\text { Summarization of } \\
\text { Opinions in Reviews" }\end{array}$ & $\begin{array}{l}\text { Hybrid Approach } \\
\text { \& Product } \\
\text { Reviews }\end{array}$ & $\begin{array}{l}\text { Two sets of } \\
\text { labeled data: } \\
\text { one for the } \\
\text { restaurant } \\
\text { domain and } \\
\text { the other for } \\
\text { the hotel } \\
\text { domain. }\end{array}$ & $\begin{array}{l}\text { Manual } \\
\text { Readability } \\
\text { Correctness } \\
\text { Completeness } \\
\text { Compactness }\end{array}$ & $\begin{array}{l}\text { Readability } 3.75 \\
\text { Correctness }=3.58 \\
\text { Completeness }= \\
3.58 \\
\text { Compactness }= \\
3.72\end{array}$ & $\begin{array}{l}\text { Takes less number } \\
\text { of aspects but } \\
\text { require huge } \\
\text { amount of training } \\
\text { data to learn the } \\
\text { ordering of aspects. }\end{array}$ \\
\hline 8 & $\begin{array}{l}\text { Genest, P. E. Et al. } \\
(2010), \text { "Text Gen- } \\
\text { eration for Abstrac- } \\
\text { tive Summarization" }\end{array}$ & $\begin{array}{l}\text { Structural } \\
\text { Approach \& } \\
\text { Single document }\end{array}$ & NA & $\begin{array}{l}\text { pyramid and } \\
\text { overall } \\
\text { responsivenes } \\
\text { s scores }\end{array}$ & $\begin{array}{l}\text { Pyramid score - } \\
29, \\
\text { Overall response - } \\
33\end{array}$ & \\
\hline 9 & $\begin{array}{l}\text { Genest, P. E et al. } \\
(2011) \text {, "Framework } \\
\text { for Abstractive } \\
\text { Summarization } \\
\text { using Text-to-Text } \\
\text { Generation" }\end{array}$ & $\begin{array}{l}\text { Structural } \\
\text { Approach \& } \\
\text { Single document }\end{array}$ & NA & $\begin{array}{l}\text { pyramid, } \\
\text { linguistic } \\
\text { quality and } \\
\text { overall } \\
\text { responsivenes } \\
\text { s scores }\end{array}$ & $\begin{array}{l}\text { Pyramid score - } \\
29, \text { Overall } \\
\text { response - 29, } \\
\text { Linguistic quality } \\
-33\end{array}$ & \\
\hline 10 & $\begin{array}{l}\text { Khan, A. et al. } \\
(2015) \text {, "A framework }\end{array}$ & $\begin{array}{l}\text { Structural } \\
\text { Approach \& }\end{array}$ & DUC - 2002 & $\begin{array}{l}\text { Pyramid score } \\
\text { \& Average }\end{array}$ & $\begin{array}{l}\text { Pyramid score } \\
0.50, \quad \text { Average }\end{array}$ & \\
\hline
\end{tabular}


Deepak Sahoo et al., International Journal of Emerging Trends in Engineering Research, 8(10), October 2020, 7294 - 7304

\begin{tabular}{|c|c|c|c|c|c|c|}
\hline & $\begin{array}{l}\text { for } \\
\text { multi-document } \\
\text { abstractive sum } \\
\text { marization based } \\
\text { on semantic role } \\
\text { labelling" }\end{array}$ & Multi document & & precision & precision -0.70 & \\
\hline 11 & $\begin{array}{l}\text { Ren, F. J. (2005), } \\
\text { "Automatic } \\
\text { Abstracting } \\
\text { Important } \\
\text { Sentences" }\end{array}$ & $\begin{array}{l}\text { Statistical and } \\
\text { Structural } \\
\text { Approach \& } \\
\text { Single document }\end{array}$ & NA & Manual & $\begin{array}{l}\text { Extraction } \\
\text { Percentage - } 75 \%\end{array}$ & \\
\hline 12 & $\begin{array}{l}\text { Katja } \\
\text { Filippova (2010), } \\
\text { "Multi-sentence } \\
\text { compression: Finding } \\
\text { shortest paths in } \\
\text { word graphs" }\end{array}$ & $\begin{array}{l}\text { Graph Based \& } \\
\text { Multiple } \\
\text { Sentences }\end{array}$ & $\begin{array}{l}\text { news } \\
\text { articles } \\
\text { presented in } \\
\text { clusters on } \\
\text { Google } \\
\text { News }\end{array}$ & $\begin{array}{l}\text { Manual } \\
\text { evaluation by } \\
\text { human } \\
\text { judges. }\end{array}$ & $\begin{array}{l}\text { grammaticality } \\
\text { and informativity } \\
\text { scores on } \\
\text { three-point likert } \\
\text { scale } \\
\text { for English and } \\
\text { Spanish } \\
(1.44 / 1.25 \text { and } \\
1.30 / 1.25)\end{array}$ & \\
\hline 13 & $\begin{array}{l}\text { Ibrahim F. Moawad et } \\
\text { al. (2012), "Semantic } \\
\text { Graph Reduction } \\
\text { Approach } \\
\text { for Abstractive } \\
\text { Text Summarization" }\end{array}$ & $\begin{array}{l}\text { Graph Based \& } \\
\text { Single document }\end{array}$ & NA & $\begin{array}{l}\text { Through case } \\
\text { studies }\end{array}$ & NA & \\
\hline 14 & $\begin{array}{l}\text { Song et al. (2005), } \\
\text { Toward Abstractive } \\
\text { Summarization Using } \\
\text { Semantic } \\
\text { Representations }\end{array}$ & $\begin{array}{l}\text { Discourse and } \\
\text { Rhetorical Based } \\
\text { Approach \& } \\
\text { Single document } \\
\text { \& Single } \\
\text { document }\end{array}$ & $\begin{array}{l}\text { Two test } \\
\text { data set } \\
\text { from Korea } \\
\text { Institute of } \\
\text { Science and } \\
\text { Technology } \\
\text { Information }\end{array}$ & $\begin{array}{l}\text { Human } \\
\text { judges }\end{array}$ & NA & \\
\hline 15 & $\begin{array}{l}\text { Carenin et al. (2013), } \\
\text { "Multidocument } \\
\text { summarization } \\
\text { of evaluative text" }\end{array}$ & $\begin{array}{l}\text { Discourse and } \\
\text { Rhetorical Based } \\
\text { Approach \& } \\
\text { Multi document } \\
\text { \& Multidocument }\end{array}$ & DUC & $\begin{array}{l}\text { Manual } \\
\text { mated } \\
\text { and } \\
\text { Automated }\end{array}$ & $\begin{array}{l}\text { Grammatical, } \\
\text { Non-redundancy, } \\
\text { Recall } \\
\text { Precision }\end{array}$ & $\begin{array}{l}\text { This approach } \\
\text { requires a set of } \\
\text { hand-crafted } \\
\text { features for each } \\
\text { product which is } \\
\text { not scalable. }\end{array}$ \\
\hline 16 & $\begin{array}{l}\text { Gerani et al. (2014) } \\
\text { "Abstractive } \\
\text { Summarization } \\
\text { of Product Reviews } \\
\text { Using Discourse } \\
\text { Structure" }\end{array}$ & $\begin{array}{l}\text { Discourse and } \\
\text { Rhetorical Based } \\
\text { Approach }\end{array}$ & & $\begin{array}{l}\text { Pairwise } \\
\text { preference by } \\
\text { crowd } \\
\text { sourcing } \\
\text { (Manual) }\end{array}$ & $\begin{array}{l}\text { Preference user } \\
\text { one } 71 \% \\
\text { Preference user } \\
\text { two } 69 \%\end{array}$ & \\
\hline 17 & $\begin{array}{l}\text { Genest et al. (2012), } \\
\text { "Fully Abstractive } \\
\text { Approach to guided } \\
\text { summarization" }\end{array}$ & $\begin{array}{l}\text { Template Based } \\
\& \text { multi- } \\
\text { document }\end{array}$ & $\begin{array}{l}\text { Guided } \\
\text { summarizati } \\
\text { on task at } \\
\text { TAC }\end{array}$ & $\begin{array}{l}\text { Pyramid, } \\
\text { (pyramid is a } \\
\text { content } \\
\text { metric) }\end{array}$ & $\begin{array}{l}\text { Pyramid score } \\
0.54\end{array}$ & $\begin{array}{l}\text { You must define } \\
\text { schemes and its } \\
\text { extraction rules for } \\
\text { different domains } \\
\text { which is not } \\
\text { scalable. }\end{array}$ \\
\hline 18 & Opinosis by Gane- & Graph Based \& & Document & Automatic & Rouge1-0.330, & This method is \\
\hline
\end{tabular}




\begin{tabular}{|l|l|l|l|l|l|}
\hline san et al. (2010), & $\begin{array}{l}\text { Reviews } \\
\text { of a product }\end{array}$ & $\begin{array}{l}\text { containing } \\
\text { reviews of a } \\
\text { query }\end{array}$ & $\begin{array}{l}\text { (ROUGE } \\
\text { score }) \\
\text { and } \\
\text { Manual } \\
\text { (readability } \\
\text { test) }\end{array}$ & $\begin{array}{l}\text { Average } \\
\text { readability score }= \\
0.67\end{array}$ & $\begin{array}{l}\text { more extractive } \\
\text { than abstractive and } \\
\text { does not validate } \\
\text { the grammatical } \\
\text { correctness of } \\
\text { sentence. }\end{array}$ \\
\hline
\end{tabular}

\section{EVALUATION AND RESEARCH ISSUES IN AUTOMATIC TEXT SUMMARIZATION}

The manual evaluation of a summarization system is a challenging task. Manual evaluation of summary is costly, time taking and likely to suffer from human variability. Therefore, automatic evaluation of summarization system gained popularity among researchers.

Different researchers proposed different automatic evaluation techniques $[61,62,63,64]$ for summary evaluation. Pyramid score [61] and ROUGE score [62] are automatic summary evaluation techniques that considers $\mathrm{N}$ gram lexical overlapping between system generated summary and human created summary for comparison.

Other than any summary evaluation method, ROUGE toolkit has gained popularity and become standard automatic machine generated summary evaluation technique.

\section{CONCLUSION}

The growth of data and information is very high due to the World-Wide-Web, therefore, there is a high demand to design and develop an efficient and accurate summarization system. Research on automatic text summarization started 50 years back but still a long way to go in this area. In initial days, research on text summarization started with summarizing the research and scientific articles then it shifted to news articles, advertisements, product reviews, electronic mail messages, and blogs. There are two approaches of text summarization that is Extractive and abstractive, and researchers tried both the approach based on the application.

The biggest challenge for text summarization is to extract gist of text from number textual sources for a user. The summarizer should produce a fruitful summary in less time and with least redundancy. This survey emphasizes the abstractive summarization approach. Usually, abstractive summarization requires a-lot-of engineering for language generation and is difficult to replicate or extend to broader domains.

\section{REFERENCES}

1. Changala R., Rajeswara Rao D, 'A survey on development of pattern evolving model for discovery of patterns in text mining using data mining techniques, Journal of Theoretical and Applied Information Technology, vol. 95, no. 16, pp.3974-3981. 2017.

2. C. D. Manning and H. Schutze. 2002. Foundations of statistical natural language processing. The MITPress, London.

3. Madhavi K. Ganapathiraju, "Overview of summarization methods", 11-742: Self-paced lab in Information Retrieval, November 26, 2002.

4. Klaus Zechner, "A Literature Survey on Information Extraction and Text Summarization", Computational Linguistics Program, Carnegie Mellon University, April 14, 1997.

5. Berry Michael W., "Automatic Discovery of Similar Words", Survey of Text Mining: Clustering, Classification and Retrieval, Springer Verlag, New York, LLC, pp. 24-43, 2004.

6. Farshad Kyoomarsi, Hamid Khosravi, Esfandiar Eslami and Pooya Khosravyan Dehkordy, "Optimizing Text Summarization Based on Fuzzy Logic", In proceedings of Seventh IEEE/ACIS International Conference on Computer and Information Science, IEEE, University of Shahid Bahonar Kerman, UK, pp.347-352, 2008.

7. H. P. Luhn, "The Automatic Creation of Literature Abstracts", Presented at IRE National Convention, New York, pp. 159-165, 1958.

8. H. P. Edmundson.," New methods in automatic extracting", Journal of the ACM, vol. 16, no. 2, pp. 264-285, April 1969.

9. Ronald Brandow, Karl Mitze, and Lisa F. Rau. "Automatic condensation of electronic publications by sentence selection". Information Processing and Management", vol. 31, no. 5, pp. 675-685, 1995. 
10. J. Kupiec, J. Pedersen, and F. Chen, "A trainable document summarizer", In Proceedings of the 18th ACM-SIGIR Conference, pp.68-73, 1995.

11. Salton, G., Singhal, A., Mitra, M., \& Buckley, C., "Automatic text structuring and summarization", Information Processing \& Management, vol. 33, no. 2, pp. 193-207, 1997.

12. Marcu, D, "The rhetorical parsing of natural language texts", In Proceedings of the $35^{\text {th }}$ Annual Meeting of the Association for Computational Linguistics and Eighth Conference of the European Chapter of the Association for Computational Linguistics, pp. 96-103, 1997.

13. R. Barzilay and M. Elhadad. "Using lexical chains for text summarization", In Proc. of the Intelligent Scalable Text Summarization Workshop (ISTS'97), ACL, 1997.

14. Radev, D. R., Jing, H., \& Budzikowska, M. "Centroidbased summarization of multiple documents: sentence extraction, utility-based evaluation, and user studies", In Proceedings of the 2000 NAACLANLPWorkshop on Automatic summarization, vol. 4, pp. 21-30, 2000.

15. Erkan, G. and Radev, D. R., "LexRank: Graph-based lexical centrality as salience in text summarization", J. Artif. Intell. Res. (JAIR), vol. 22, no. 1, pp. 457-479, 2004.

16. Erkan, G. and Radev, D. R.,” LexPageRank: Prestige in Multi-Document Text Summarization", In EMNLP, vol. 4, pp. 365-371, 2004.

17. Radev, D. R., McKeown, K. R. "Generating Natural Language Summaries from Multiple On-Line Sources", In Proceedings of Association for computational linguistics, vol. 24 , no. 3 , pp. 470-500, 1998.

18. Genest, P. E., Lapalme, G. "Fully Abstractive Approach to Guided Summarization", Association for Computational Linguistics, pp. 354-358, 2012.

19. Genest, P. E., Lapalme, G. "Text Generation for Abstractive Summarization", In Proceedings of the third text analysis conference, National Institute of Standards and Technology, 2010.

20. Genest, P. E., Lapalme, G. "Framework for Abstractive Summarization using Text-to-Text Generation", In Proceedings of the Workshop on
Monolingual Text-To-Text Generation, Association for Computational Linguistics, pp. 64-73, 2011.

21. DA kumar, ASCS Sastry, PVV kishore and EK kumar, "Indian sign language recognition using graph matching on 3D motion captured signs", Multimedia tools and applications, 2018.

22. Das S.K., Nayak S.R., Mishra J., "Fractal geometry: The beauty of computer graphics", Journal of Advanced Research in Dynamical and Control Systems, vol. 9, no. 10, pp.76-82, 2017

23. Ramprasad C., Varma P.L.N., Satyanarayana S., Srinivasarao N., "Morphism of m-Polar Fuzzy Graph", Advances in Fuzzy Systems, 2017.

24. Barzilay, R., Lee, L. "Learning to paraphrase: an unsupervised approach using multiple-sequence alignment", In Proceedings of the Association for Computational Linguistics on Human Language Technology, Morristown, NJ, USA, pp.16-23, May 2003.

25. Barzilay, R., McKeown, K.R., "Sentence fusion for multidocument news summarization", Computational Linguistics, vol. 3, no. 3, pp. 297-328, 2005.

26. Ganesan, K., Zhai, C., Han, J. "Opinosis: a graphbased approach to abstractive summarization of highly redundant opinions", In Proceedings of the 23rd International Conference on Computational Linguistics, pp. 340-348, 2010.

27. Filippova, K., "Multi-sentence compression: Finding shortest paths in word graphs", In Proceedings of the 23rd International Conference on Computational Linguistics, pp. 322-330, Aug 2010.

28. Moawad, I. F., \& Aref, M., "Semantic graph reduction approach for abstractive Text Summarization", In Computer Engineering \& Systems (ICCES), pp. 132-138, 2012.

29. Kumar, Niraj, Kannan Srinathan, Vasudeva Varma. “ A knowledge induced graph-theoretical model for extract and abstract single document summarization", International Conference on Intelligent Text Processing \& Computational Linguistics, pp.408-423, 2013.

30. Liu, F., Flanigan, J., Thomson, S., Sadeh, N., \& Smith, N. A., "Toward abstractive summarization using semantic representations", In Proceedings of the 2015 Conference of the North American Chapter of the 
Association for Computational Linguistics: Human Language Technologies, pp. 1077-1086, 2015.

31. Banarescu, L., Bonial, C., Cai, S., Georgescu, M., Griffitt, K., Hermjakob, U., ... \& Schneider, N., "Abstract meaning representation for sembanking", In Proceedings of the 7th Linguistic Annotation Workshop and Interoperability with Discourse pp. 178186, 2013.

32. Carenini, G., Cheung, J. C. K., Pauls, A. "Multidocument summarization of evaluative text", Computational Intelligence, vol. 29, no. 4, pp.545-576, 2013.

33. Reiter E, Dale R. "Building natural language generation systems", Cambridge university press, Jan 2000.

34. Gerani, S., Mehdad, Y., Carenini, G., Ng, R. T., Nejat, B.: "Abstractive Summarization of Product Reviews Using Discourse Structure", In Proceedings of Empirical Methods on Natural Language Processing, pp. 1602-1613, 2014

35. Daniel Marcu, "The rhetorical parsing of natural language texts", Proc. 35th Annual Meeting of the Association for Computational Linguistics, 1997.

36. Daniel Marcu, "The rhetorical parsing of unrestricted texts: A surface-based approach", Computational Linguistics, vol. 26, no. 3, pp. 395-448, 2000.

37. K. Ono, K. Sumita and S. Miike, "Abstract generation based on rhetorical structure extraction", Proc. Int. Conf. on Computational Linguistics, 1994.

38. Teufel, Simone and Marc Moens, "Summarizing scientific articles: Experiments with relevance and rhetorical status", Computational Linguistics, vol. 28, no. 4, pp. 409-445, 2002.

39. Y. L. Lee and K. S. Choi, "Automatic text summarization using rhetorical structure", Proc. 11th Conf. on Hangul and Korean Information Processing, 1999.

40. Song, S. K., Jang, D. H., \& Myaeng, S. H., "Text summarization based on sentence clustering with rhetorical structure information". International Journal of Computer Processing of Oriental Languages, 18(02), pp. 153-170, 2005.
41. Di Fabbrizio, G., Stent, A. J., Gaizauskas, R. "A Hybrid Approach to Multi-document Summarization of Opinions in Reviews", INLG 2014, pp. 54-63, 2014.

42. Mann, W. C., Thompson, S. A. "Rhetorical structure theory: A theory of text organization", University of Southern California, Information Sciences Institute, pp. 87-190, 1987.

43. Le, H. T., \& Le, T. M., "An approach to abstractive text summarization", In Soft Computing and Pattern Recognition (SoCPaR), pp. 371-376, 2013.

44. Genest, P. E., \& Lapalme, G., "Text Generation for Abstractive Summarization". In TAC, November 2010 .

45. Genest, P. E., \& Lapalme, G., "Framework for abstractive summarization using text-to-text generation", In Proceedings of the Workshop on Monolingual Text-To-Text Generation pp. 64-73, June 2011.

46. Khan, A., Salim, N., \& Kumar, Y. J., "A framework for multi-document abstractive summarization based on semantic role labeling", Applied Soft Computing, vol. 30, pp. 737-747, 2015.

47. Ren, F. J., "Automatic abstracting important sentences" International Journal of Information Technology \& Decision Making, vol. 4, no. 01, pp.141152,2005

48. Bing, L., Li, P., Liao, Y., Lam, W., Guo, W., \& Passonneau, R. J., "Abstractive multi-document summarization via phrase selection and merging", arXiv preprint arXiv:1506.01597, 2015.

49. Zhang, J., Cheng, X., Wu, G., \& Xu, H., "AdaSum: an adaptive model for summarization", In Proceedings of the 17th ACM conference on Information and knowledge management (pp. 901-910). ACM, Oct 2008 .

50. AK Bashir, R Arul, S Basheer, G Raja, R Jayaraman, NMF Qureshi, "An optimal multitier resource allocation of cloud RAN in 5G using machine learning”, Transactions on Emerging Telecommunications technologies, 2019.

51. Ayushree Arora, Sandeep Kumar, "Comparative Analysis of aodv and dsdv using machine learning approach in manet", Journal of engineering science and technology, 2017.

52. Prasanth Y., Grace Hepsiba M., Mounika T., Pavan Kumar T., Raghavendra Kumar G., "Application of machine learning techniques on naval and 
telecommunication system failure data”, Journal of Advanced Research in Dynamical and Control Systems,9(Special Issue 6), PP.1065-1076, 2017.

53. Kotkar V.A., Sucharita V., "A comparative analysis of machine learning based anomaly detection techniques in video surveillance", Journal of Engineering and Applied Sciences,12(Specialissue12), pp.9376-9381, 2017.

54. Rao K.V.S.N.R., Battula S.K., Krishna T.L.S.R., “A smart heuristic scanner for an intrusion detection system using two-stage machine learning techniques', International Journal of Advanced Intelligence Paradigms, pp.519-529, 2017.

55. Gross, O., Doucet, A., \& Toivonen, H. (2014, July). "Document summarization based on word associations". In Proceedings of the 37th international ACM SIGIR conference on Research \& development in information retrieval, pp. 1023-1026. July 2014.

56. Rush, A. M., Chopra, S., \& Weston, J., "A neural attention model for abstractive sentence summarization. arXiv preprint arXiv:1509.00685, 2015.

57. Nallapati, R., Zhou, B., Gulcehre, C., \& Xiang, B.. "Abstractive text summarization using sequence-tosequence rnns and beyond", arXiv preprint arXiv:1602.06023. 2016.

58. I Srivani, GSV Prasad, DV Ratnam, “A Deep Learning-Based Approach to Forecast Ionospheric Delays for GPS Signals", IEEE Geoscience and remote sensing letters, 2019.

59. Reddy A.V.N., Phanikrishna C., "Contour tracking based knowledge extraction and object recognition using deep learning neural networks", Proceedings on 2016 2nd International Conference on Next Generation Computing Technologies, NGCT 2016, pp..352-354, 2017.

60. Chopra, S., Auli, M., \& Rush, A. M. "Abstractive sentence summarization with attentive recurrent neural networks", In Proceedings of the 2016 Conference of the North American Chapter of the Association for Computational Linguistics: Human Language Technologies (pp. 93-98). 2016.

61. Nenkova, A., Passonneau, R., \& McKeown, K., "The pyramid method: Incorporating human content selection variation in summarization evaluation, ACM Transactions on Speech and Language Processing (TSLP), vol. 4, no. 2, 2007.

62. Lin, C. Y., \& Hovy, E. (2003, May). "Automatic evaluation of summaries using n-gram coccurrence statistics", In Proceedings of the 2003 Conference of the North American Chapter of the Association for Computational Linguistics on Human Language Technology. Association for Computational Linguistics. vol. 1, pp. 71-78, May 2003.

63. Hovy, E., Lin, C. Y., \& Zhou, L., "Evaluating duc 2005 using basic elements", In Proceedings of DUC, Oct 2005.

64. Hovy, E., Lin, C. Y., Zhou, L., \& Fukumoto, J.. "Automated summarization evaluation with basic elements", In Proceedings of the Fifth Conference on Language Resources and Evaluation, pp. 604-611. May 2006.

65. Sahoo, D. and Balabantaray, R.C., "A Hybrid Approach to Retrieve Knowledge from a Document", International Journal of Knowledge Management (IJKM), vol. 16, no. 1, pp.83-100. 2020.

66. Sahoo, D., Bhoi A. and Balabantaray, R.C., "Hybrid Approach to Abstractive summarization", Procedia computer science, vol. 132, pp.1228-1237. 2020.

67. M. V. D prasad, M. teja kiran kumar, K.H.S. Sharma, M. gopal, R. kumari, Sk hassane, "Human activity recognition using Deep Learning", IJETER, vol. 7, no. 11, 2019.

68. S. inthiyaz, M. V. D prasad, R. usha, N.T.B sri sai, P. pavan kumar, Sk hassane, "Agriculture based plant leaf health assessment tool: A Deep learning perspective", IJETER, vol. 7, no. 11, 2019. 